\title{
Análisis lanimétricos de camélidos: una herramienta metodológica
}

Plinio Gechle C. (*), Makia Anjonia Benavente A. (**), Carlos lhomas W. (***), y José M. Benavente H. (***).

\section{RESUMEN}

En el presente estudio se exponen los resultados del análisis estadístico de los promedios de diámetro realizado a las inuestras de lana de animales actuales y las conclusiones de su uso como patrones para contrastación arqueológica. Además se entrega un listado de las muestras arqueológicas estudiadas y su identificación, en base a análisis lanimétricos macro y microscópicos.

\section{ABSTRACT}

The present study points out the results of the statistical analyses of the diameter means taken from wool samples of modern animals, as well as the conclusions concerning their use as patterns for archaeological verification. In addition, a list of the archaeological samples studied is delivered with its identification based on macro and microscopical lanimetric analyses.

\section{Introducción}

l.os estudios de camélidos andinos (Adaro, I. Benavente M. A., 1998, Benavente M. A. 19971998, Herrera, O., 1998, Menegaz; Salamme, M. y Ortiz, 1998. Cartajena, I.;Benavente, M. A.; Gecele, D.: Concha y Benavente, J. M., 1999) en estas últi-

(*) Iniversidad Santo Tomás. Escuela de Medicina Veterinaria.

(*1) Universidad de Chile. Departamento de Antropologia.

(***) Universidad de Chile. Facultad de Economía. mas décadas han cobrado especial importancia en las investigaciones de naturaleza arqueológica. Estos han sido desarrollados por diversos investigadores, tanto nacionales como extranjeros. Este trabajo se inserta dentro del período denominado Formativo para el Norte Grande de Chile, específicamente la zona del Loa Medio. (Proyecto FONDECY'T No 1970537 a cargo de los autores de este artículo).

El objetivo general de nuestro estudio ha sido determinar, a través del análisis tanto de patrones como de los restos arqueofaunísticos, la función zoolécnica específica a que lueron sometidos dichos animales. Por tanto uno de los temas importantes a trabajar fueron los fanéreos pertenecientes a la Familia Camelidae, y en especial, referido a Llama.

Como nuestro enfoque tiene dos vertientes, una biológica y otra arqueológica, nuestra tarea se abocó en una primera instancia a la determinación de rasgos e indicadores relativos al análisis de patrones de camélidos actuales y por otro al análisis de los restos arqueológicos referidos a fanéreos del sitio de Topater. (Serracino, 1994 ms.; Thomas el al 1997).

El sitio Topater corresponde a un cementerio localizado en el área de Calama, el que por la naturaleza de sus contextos correspondería a grupos caravaneros procedentes de tierras altas, selvas occidentales y Tarija. Dicho grupo se habría especializado en el trálico de objetos rituales. El resultado de este plantcamiento sería que las poblaciones habrían utilizado al animal en forma diversificada, en lo que respecta a su función zootécnica.

\section{Material y método}

Para el presente estudio, se obtuvieron 4 especímenes de llama con las siguientes características:

Animal 1: Hembra vieja, sobre 4 años, utilizada como animal de carga.

Animal 2: Macho adulto, 3 años, entero, utili- 
zado como reproductor y con aptilud lanera.

Animal 3: Macho guarizo joven, de dos años. entero.

Animal 4: Macho capón joven, menor de dos aก̃os.

Siguiendo las indicaciones de Benavente, $\Lambda$., y col.(1993) en su manual de "Contribución a la deerminación de especies animales en arqueología: Familia Camelidac y Taruca del Norte", se obuvieren mucstras de lana por eorte con tijori en 10 zonas corporales en cala animal y se reatizaron los análisis lanimétricos, obleniéndose los valores de diámetro promedio por anmal y zona corporal, para los animakes actuales.

Las zonas corporales consideradas son las siguientes:

Zona 1: Región femoral (rodilla)

Zona 2 : Región torácica lateral, tercio inferior (vientre)

Zona 3: Región cubital (codo)

Zona 4: Región de la articulación coxal (pierna)

Zonat 5: Arco costal, tercio medio (1lanco)

Zona 6: Región escapular ( paleta)

Zona 7 : Región sacral dorsal (anca)

7.ona 8: Región dorsal torácica, nivel de última costilla (espalda)

Zona 9: Región dorsal de tórax, en línea interciscapular (cruz)

Zona 10: Región dorsal del cuello (cuello)

De cada muestra original de lana se realizó un examen laniméurico microscópico, obteniéndose una porción que fue montada y fijada en un portaobjeto con Bálsamo de Canadá. Se observaron en un lanámetro con aumento de $400 \mathrm{x}$, a fin de determinar la finura promedio scgún las nomas de la British Standard Institution: " Determination of wool fibre lineness by the use of a Projection Microscope".

Para las mucstas arqucológicas, se seleccionó un total de 46 colecciones procedentes de los contextos de fanéreos correspondientes al sitio topater, entre los cuales destacan vellones, pelos de patas, cordelería, restos de cueros y tejidos. Las muestras lueron limpiadas y desgrasadas, para luego ser montadas en portaobjetos, siguiendo la mctodología ya descrita. Dadas las características de las muestras, y los resultados previos de las mediciones de diámetros o finuras, se readizó un análisis lanimétrico, tanto macrosé́pico como microscópico, de contrastación con los patrones ya preestablecidos.

\section{Diámetro de filora. Análisis estadístico}

Una de las características lanimétricas más importantes en las fibras animales, es su diámetro. Este detemina su uso textil, es un rasgo fácil de medir y existe cierta uniformidad intraespecic.

lin este acápite se presenta la metodología que fué utilizada para la connastación de las hipótesis planteadas. Esta descansa fuertemente en el contraste estadístico de hipótesis sobre diferencias de medias poblaciomales, utilizando muestras obtenidas de animales acuales.

\subsection{P'rueba de Hipótesis sobre Medias Pobla- cionales}

\section{Caso : 2 muestras}

Sean $X_{1}, X_{2}, \ldots . X_{n 1}$ y $Y_{1}, Y_{2}, \ldots . Y_{n y}$ muestras adeatorias provenientes de des distribuciones normatles independientes con media $\mu_{x}$ y $\mu_{y}$ y variauras $\sigma^{2}$, y $\sigma^{2}$ respectivanente.

Suponga que se desea probar

$$
H_{0}: \mu_{x}-\mu_{y}=0
$$

contra

$$
\mathrm{H}_{1}: \mu_{\mathrm{x}}-\mu_{\mathrm{y}} \neq 0
$$

Iis decir, que las medias de am bas poblaciones no presentan diferencias significativas desde un punto de vista estadístico.

Se sabe que los mejores estimadores de las medias de poblaciones y sus varianzas son las medias y varianzas muesuales. Por otra parte si las varianzas poblacionales son desconocidas pero iguales ${ }^{2}$ en-

1 Se asme que los procesos aleatorios que están detrás de los datos sigue una distribución Normal. Sin embargo, la distribución t-student es muy robusta o insensible a la suposicion de nomalidad. en particular si el tamaño de la muestra es mayor a 15 [Canavos $1988: 338]$.

2 A pesar que si se sospecha que las varianzas asociadas a cada muestra no son iguales, se ha demostrado que si nx y ny son relativamente parecidos, la distribución t-student es robusta o insensible a este supuesto [Scheflé 1959]. 
tonces, se definc el estadístico:

$$
T c=\frac{X-Y-0}{S p \sqrt{\frac{1}{n_{2}}+\frac{1}{n_{y}}}} \sim \text { t-Student }_{n+n+y_{-2}}
$$

Donde:

$X$ : Media muestral de una de las muesuas

$\mathrm{Y}$ : Media muestral de la otra muestra

$S_{\mathrm{p}}$ : Desviación estándar común para las muestras, definida de la siguiente manera:

$$
S_{p}^{2}=\frac{\left[\left(n_{i}-I\right) S^{2}+\left(n_{y}-I\right) S_{y}^{2}\right]}{n_{x}+n_{y}-2}
$$

Donde:

$\mathrm{S}_{\mathrm{p}}^{2}$ : Varianza de la muestra $\mathrm{x}$

$S^{2}:$ : Varianza de la muestra y

Ln consecuencia, el criterio de rechazo de la hipótesis nula on favor de que efectivamente existen diferencias signilicativas, desde un punto de vista estadístico, entre las dos medias poblacionales, será :

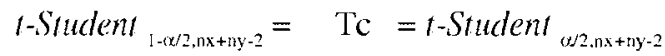

\subsection{Muestra utilizada}

LLAMA I HEMBRA SOBRE

\begin{tabular}{|c|c|c|c|c|c|c|c|c|c|c|}
\hline & & & 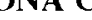 & 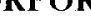 & & & & & & \\
\hline & 1 & 2 & 3 & 4 & 5 & 6 & 7 & 8 & 9 & 10 \\
\hline $\begin{array}{l}\text { Número de } \\
\text { Observaciones }\end{array}$ & 100 & 100 & 100 & 100 & 100 & 100 & 100 & 100 & 100 & 100 \\
\hline Diámetro Medio & 36,40 & 34,30 & 39,38 & 35,72 & 33,78 & 36,96 & 36,72 & 33.66 & 38,98 & 30,28 \\
\hline $\begin{array}{l}\text { Desviación } \\
\text { Estándar }\end{array}$ & 6,31 & 4,98 & 7,66 & 6,22 & 5,88 & 6,19 & 4,96 & 5,83 & 4,70 & 4,29 \\
\hline
\end{tabular}

4 AÑOS, CARGUERA

LLAMA 2 MACHO

LANERO 3 ANNOS

ZONA CORPORAL

$\begin{array}{ccccccccccc} & 1 & 2 & 3 & 4 & 5 & 6 & 7 & 8 & 9 & 10 \\ \text { Número de } & 100 & 100 & 100 & 100 & 100 & 100 & 100 & 100 & 100 & 100\end{array}$

Observaciones

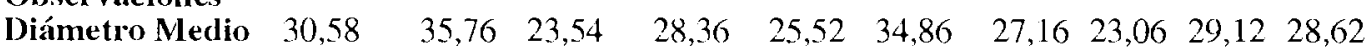

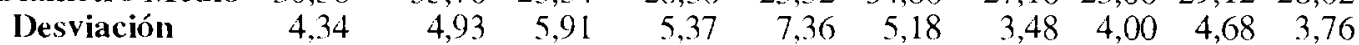

Estándar

LLAMA 3

MACHO GUARIZO

Número de

$12 \begin{array}{ccc}\text { ZONA CORPORAL } \\ 3\end{array}$

Observaciones

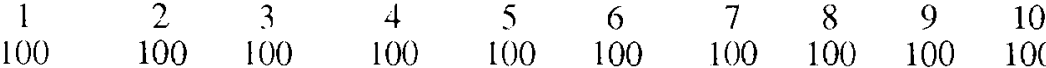

Diámetro Medio

Desviación

Estándar

$\begin{array}{crrrrrrrrr}32,7 & 34,54 & 35,8 & 26,98 & 31,8 & 33,02 & 28,78 & 32,18 & 26,36 & 33,98 \\ 4,85 & 3,77 & 6,49 & 3,03 & 4,52 & 4,38 & 2,87 & 3,42 & 3,49 & 3,51\end{array}$

LLAMA 4 MACHO

CAPON IOVEN

Número de

ZONA CORPORAL

Observaciones

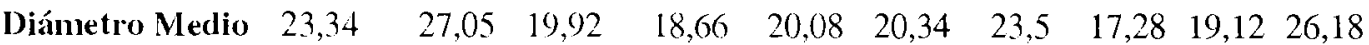

Desviación

5,15

4,00

$3,02 \quad 4,07 \quad 3,60$

$4,60 \quad 3,60 \quad 3,51 \quad 3,90$

Estándar 


\section{Resultados}

L.os resultados de la contrastación sobre diferencias entre las medias poblacionales, se presentan a continuación. Los valores que se muestran son el valor cncontrado para el estadístico de prueba, al comparar las zonas de a pares. Considerando un nivel de crror tipo I de un $5 \%$. para que la hipótesis nula de no diferencia entre medias sea rechazada en lavor de yue existe diferencia entre éstas con un $95 \%$ de contianzal, el valor obtenido deberá serr mayor al valor crítico de tabla, el cual es de 1.65 .

LLAMA I

HEMBRA SOBRE 4 AÑNOS, CARGUERA

ZONAS CORPORAIJS

\begin{tabular}{|c|c|c|c|c|c|c|c|c|c|c|}
\hline ZONA & 1 & 2 & 3 & 4 & 5 & 6 & 7 & 8 & 9 & 10 \\
\hline$I$ & & 10,18 & -7.97 & 1,92 & 7.51 & $-1,58$ & $-0,95$ & 7,87 & $-7,78$ & 18,80 \\
\hline 2 & & & $-18,00$ & -8.19 & $-2,43$ & $-11,91$ & $-11,86$ & $-2,07$ & $-19,28$ & 8,87 \\
\hline 3 & & & & 9,83 & 15,22 & 6,50 & 7,49 & 15,58 & 1,14 & $.26,32$ \\
\hline 4 & & & & & 5,58 & $-3,52$ & $-2,99$ & 5,94 & $-9,87$ & 16,78 \\
\hline 5 & & & & & & $-9,15$ & -8.93 & 0,35 & $-15,99$ & 10,97 \\
\hline 6 & & & & & & & 0,72 & 9,52 & $-6,12$ & 20,63 \\
\hline 7 & & & & & & & & 9.32 & $-7,27$ & 21,17 \\
\hline 8 & & & & & & & & & $-16,40$ & 10,63 \\
\hline 9 & & & & & & & & & & $-3,87$ \\
\hline 10 & & & & & & & & & & \\
\hline
\end{tabular}

LLAMA 2

MACHO LANERO 3 AÑOS

ZONAS CORPORALES

\begin{tabular}{|c|c|c|c|c|c|c|c|c|c|c|}
\hline ZONA & $I$ & 2 & 3 & 4 & 5 & 6 & 7 & 8 & 9 & 10 \\
\hline$I$ & & $-17,18$ & 21,98 & 7,12 & 14,79 & $-13,87$ & 12,23 & 26,03 & 4,86 & 6,89 \\
\hline 2 & & & 37,12 & 23,06 & $2^{9}, 22$ & 2.83 & 29,66 & 42,50 & 21,42 & 24,23 \\
\hline 3 & & & & $-14,35$ & $-5,43$ & $-33,98$ & $-11,81$ & 1,52 & $-17,14$ & $-16,33$ \\
\hline 4 & & & & & 7,96 & $-20,01$ & 4,03 & 17,31 & $-2,40$ & $-0,86$ \\
\hline 5 & & & & & & $-26,37$ & $-4,98$ & 7,30 & $-10,37$ & $-9,30$ \\
\hline 6 & & & & & & & 26.16 & 38.93 & 18,28 & 20,87 \\
\hline 7 & & & & & & & & 14,98 & $-6,86$ & $-5,43$ \\
\hline 8 & & & & & & & & & $-20,56$ & $-19,96$ \\
\hline 9 & & & & & & & & & & 1,72 \\
\hline 10 & & & & & & & & & & \\
\hline
\end{tabular}




\section{ZONAS CORPORALES}

\begin{tabular}{|c|c|c|c|c|c|c|c|c|c|c|}
\hline & 1 & 2 & 3 & 4 & 5 & 6 & 7. & 8 & 9 & 10 \\
\hline$Z O N A$ & $I$ & 2 & 3 & 4 & 5 & 6 & 7 & 8 & 9 & 10 \\
\hline$I$ & & $-6,27$ & $-9,20$ & 20,38 & 2,94 & $-1,05$ & 14,11 & 1,81 & 21,96 & $-4,43$ \\
\hline 2 & & & $-3,93$ & 29,00 & 9,52 & 5,32 & 22,35 & 8,80 & 30,37 & 2,08 \\
\hline 3 & & & & 28,59 & 12,06 & 8,43 & 22,94 & 11,50 & 29,89 & 5,75 \\
\hline 4 & & & & & $-17,55$ & $-22,19$ & $-7,41$ & $-20,47$ & 2,43 & $-27,37$ \\
\hline 5 & & & & & & $-4,09$ & 11,11 & $-1,35$ & 19,23 & $-7,69$ \\
\hline 6 & & & & & & & 15,75 & 3,01 & 23,75 & $-3,42$ \\
\hline 7 & & & & & & & & $-13,55$ & 9,60 & $-20,59$ \\
\hline 8 & & & & & & & & & 22,14 & $-6,84$ \\
\hline 9 & & & & & & & & & & $-.56,09$ \\
\hline 10 & & & & & & & & & & \\
\hline
\end{tabular}

LLAMA 4

MACHOCAPON IOVEN

\section{ZONAS CORPORALES}

\begin{tabular}{|c|c|c|c|c|c|c|c|c|c|c|}
\hline & 1 & 2 & 3 & 4 & 5 & 6 & 7 & 8 & 9 & 10 \\
\hline$Z O N A$ & 1 & 2 & 3 & 4 & 5 & 6 & 7 & 8 & 9 & 10 \\
\hline 1 & & $-13,75$ & 11.74 & 16,37 & 10,74 & 10,14 & $-0,51$ & 20,49 & 14,34 & $-9,44$ \\
\hline 2 & & & 27,97 & 33,35 & 26,11 & 25.97 & 13,63 & 37,06 & 30,57 & 4,70 \\
\hline 3 & & & & 5,00 & $-0,59$ & $-1,59$ & $-12,70$ & 10,02 & 3,06 & $-23,27$ \\
\hline 4 & & & & & $-5,33$ & $-6,53$ & $-17,53$ & 5,36 & $-1,80$ & $-28,58$ \\
\hline 5 & & & & & & $-0,94$ & $-11,61$ & 10,11 & 3,49 & $-21,61$ \\
\hline 6 & & & & & & & $-11,03$ & 11,41 & 4,58 & $-21,33$ \\
\hline 7 & & & & & & & & 21,71 & 15,38 & $-9,19$ \\
\hline 8 & & & & & & & & & $-6,90$ & $-32,50$ \\
\hline 9 & & & & & & & & & & $-25,94$ \\
\hline 10 & & & & & & & & & & \\
\hline
\end{tabular}

Los resultados anteriores muestran que en general existe una marcada diferencia entre los diámetros promedios para distintas zonas del cuerpo de un mismo animal.

No obstante, las pocos casos en que es indistinguible una diferencia en diámetros se han resaltado en negrito. Por ejemplo, para el caso de la llana carguera, los datos sugieren que no existen grandes diferencias entre la Zona 1, la Zona 6 y la Zona 7, en lo que se refiere al diámetro de los pelos. 'También la Zona 3 con la Zona 9 no se observan diferencias significativas.

Al observar con más cuidado los valores de las pruebas -student realizados, se observa que las 
mayores diferencias entre las zonas aparecen al comparar la \%ona 10 con el reso de las \%onas. $\Lambda \mathrm{I}$ observar los valores de los estadigrafos de prueba para la columna IO de la llama carguera, se puede notar que estos úllimos son. con excepción de dos o tres zonas, los más altos valores cncontrados. Io anterior muestra cue, a pesar de existir diferencias entre los diámetros de las distintas zonas en el animal, la Zona 10 es radicalmente diferente al resio. En particular, el signo positivo encontrado signilical que las demás zonas tienen un diámetro promedio largamente superior.".

Parael caso del macho lancro, sólamentc la Zona 3 con la Zona 8 aparceen como similares en cuanto a los diámetros, pero son estas mismas dos zonas, al cvaluar los valores absolutos de las prucbas realizadas, las gue aparecen como las más diterentes al resto de las zonas. Sus signos positivos muestran que los diánetros de cstas zonals son mareadamente inferiores al resto.

Un fenómeno similar al de la llama carguera se observa para el caso del macho guarizo. Las Zonas 1 y 6 y zonas 1 y 8 aparecen como similares pero al comparar las \%onas 6 y 8 entre cllas, son difcrentes. I a explicación está en los signos, que al ser distintos at compararlas con la \%ona 1 , significan que la Zona

Zona 1

\begin{tabular}{|lrrr|}
\hline & Llama 1 L lama 2 Llama 3 & Llama 4 \\
L.Lama 1 & 7,60 & 4,65 & 16,04 \\
L.lama 2 & & $-13,96$ & 5,33 \\
Llama 3 & & & 21,75 \\
Llama 4 & & & \\
\hline
\end{tabular}

\section{Tona 3}

\begin{tabular}{|lcrr|}
\hline & L.lama 1 Llama 2 Llama 3 J Lama 4 \\
Llama 1 & 16,37 & 3,57 & 23,29 \\
Llama 2 & & $-3,26$ & $10,7,5$ \\
Llama 3 & & & 13,23 \\
Llama 4 & & & \\
\hline
\end{tabular}

3 Si ha lecura es en forma de columnas. un signo posilivo signilica que esta zona contienen pelos con diámetros menores a cada una de las zonas com que se la compara. Un signo negative en una lectura horizontal (en forma de lilas) significa lo mismo.
6 es marcadamente superior a la Zona 8. Adicionalmente, tanto la zona 4 como la zona 9 aparecen como significativamente distintas al resto; ta \%ona 4 como relativamente menor - dado sus signos negativos - y la Zona 9 mayor.

linalmente, para el caso del macho capón. se observa que nuevanente la mayoría de las zonas presentan, desde un punto de vista estadístico, diferencias significativas entre los diámetros de pelos. I a excepción, en este caso la constituyen las Zonas 3,5 y 6 yue aparecen bastantc similares entre cllas. Una lectura por columnas muestaa que los diámetros de latoma lo son mareadannente superiores al resto, lo mismo se obscrva para la zona 2 , al realizar una keciura horisontal.

Ba siguiente paso de la investigación trata sobre diferencias entre amimales para la mismá zona del cuerpor. lis decir, estudiar si cxisten, deste un punto de vistat cstalistico, difcrencias significativats para los diámetros de los pelos para lá misma zona pero ahora al compararlas entre distintos animales. I.os resulatdos de estass prucbas se presentell a continuatción, y deburán ser in lerpretados de la misma manera que aquellos entregaldos en los tabulados anteriores.

Comose puede observar de los cuadros anterio-

\%ona 2

\begin{tabular}{|lrrr|}
\hline & L Launa I Llama 2 L Lama 3 L Launa 4 \\
Llama 1 & $-3,97$ & $-2,50$ & 8,58 \\
Llama 2 & & 1,97 & 13,01 \\
Llama 3 & & & 12,80 \\
Llama 4 & & & \\
\hline
\end{tabular}

Zona 4

\begin{tabular}{|lcrr|}
\hline & Llama I Llama 2 Llama 3 & Llama 4 \\
Llama I & 8,96 & 12,64 & 24,68 \\
l.lama 2 & & 2,24 & 15,73 \\
Llama 3 & & & 19,45 \\
Llama 4 & & & \\
\hline
\end{tabular}


Zona 5

\begin{tabular}{|lrrr|}
\hline & Llama 1 Llama 2 Llama 3 & Llama 4 \\
Llama 1 & 8,77 & 2,67 & 19,16 \\
Llama 2 & & $-7,27$ & 6,47 \\
LLama 3 & & & 19,28 \\
Llama 4 & & & \\
\hline
\end{tabular}

Zona 7

\begin{tabular}{|lrrr|}
\hline & Llama 1 Llama 2 L Lama 3 & Llama 4 \\
Llama 1 & 15,77 & 13,86 & 19,53 \\
Llama 2 & & $-3,59$ & 6,34 \\
ILama 3 & & & 9,73 \\
LLama 4 & & & \\
\hline
\end{tabular}

Yonas

\begin{tabular}{|lrrr|}
\hline & Llama 1 Llama 2 L Lama 3 & l.lama 4 \\
Llama 1 & 14,86 & 21,56 & 33,86 \\
Llama 2 & & 4,73 & 17,09 \\
Llama 3 & & & 14,64 \\
Llama 4 & & & \\
\hline
\end{tabular}

res, lodos los valores encontrados para el estadístico de prueba son mayores que el valor crítico de tabla (i.e. 1.65 para un 95\% de contianza). Lo anterior significa que al comparar la misma zona del cuerpo entre los cuatro tipos de animales, se encuentra que clectivamente los diámetros promedios son diferentex contre animales.

No obstante lo anterior, al considerar los valores absolutos de los estadísticos de pruebal, se observa que el macho capón joven tiene diámetros promedios de pelo que son radicalmente distintos a los otros tres ejemplares. Lo anterior es particularmente notorio en las Zonas 1, 2, 4 y 6 . Mientras que en la Zonas 3 tanto el capón como el lanero son muy diferentes a los otros dos animales. Finalmente, en la Zona 7 la hembra carguera aparece con diámetros de pelos en promedio bastante mayores a las demás especies.

\subsection{Consideraciones finales}

Los resultados anteriores sugieren que los diámetros promedio de los pelos provenientes de distintas
Zoná 6

\begin{tabular}{|lrrr|}
\hline & Llama 1 Llana 2 Llama 3 & Llama 4 \\
Llama 1 & 2,60 & 5,19 & 23,21 \\
Llama 2 & & 2,71 & 23,02 \\
L.Lama 3 & & & 22,37 \\
Llama 4 & & & \\
\hline
\end{tabular}

Zona 8

\begin{tabular}{|lrrr|}
\hline & Llama 1 Llama 2 Llama 3 & Llama 4 \\
L.lama 1 & 14,99 & 2,19 & 23,91 \\
Llama 2 & & $-17,31$ & 10,73 \\
Llama 3 & & & 29,98 \\
L_lama 4 & & & \\
\hline
\end{tabular}

\%ona I0

\begin{tabular}{|lrrr|}
\hline & Llana 1 Llama 2 Llama 3 & Llama 4 \\
Llama 1 & 2,91 & $-6,67$ & 7,07 \\
Llauna 2 & & $-10,42$ & 4,51 \\
Llama 3 & & & 14,87 \\
Jlanna 4 & & & \\
\hline
\end{tabular}

zonats de un animal son estadísticamente diferentes. Estos resultados son análogos al comparar una misma zonal corpórea entre los distintos especímenes.

No obstante lo anterior, existe algunos valores encontrados en los estadísticos de prueba que sugieren que para ciertas zonas y/o para ciertos animales, existen marcadas diferencias a favor de alguna (o) que podrían sugerir un cierto patrón uniforme de diferencia inter zona y/o inter anunal.

Los resultados anteriores deben ser tratados con cautela. Primero pues sólo pueden ser adscritos a los cuatrocspecímenes considerados. Es decir, dado que no se sabe el grado de representalividad que tienen cada uno de estos especímenes, no se puede inferir que las diferencias encontradas entre por ejemplo, una llama carguera con un guarizo, sea extrapolable para todas las llamas cargueras, al compararlas con cualquier llamo guarizo.

Si se tuviera un indicador de la varianza intra especic, es decir, la variabilidad que se podria observar entre los diámetros de pelos para, por ejemplo, los machos laneros, los resultados entonces podrían ajustarse para explicar diferencias entre universos. 
Finalmente, y quizás un aspecto muy importante para los objetives gencrales de este estudio, los resultados anteriores no pueden ser utilizados para inferir diferencias entreespeciesal considerar especímenes arqucológicos. Lo anterior, no sólo porque los molivos planteados para las muesuras actuales son lambién válidos para este otro tipo de muestras, sino que además no existe evidencia suliciente que respalke la posibilidad de comparar, con algún grado de contrabilidad, muestras actuales con muestras arqueológicas.

\subsection{Análisis lanimétrico de muestras artpueológicas.}

Las fibras animales lienen caracteristicas macroscópicas y nnicroscópicás cue das hacen diferenciables entre sí y su identificáción precisal requicre del desárrollo de adecuadas lécnicás. parte de las cuales ticnen como fundamento el establecimiento de patrones.

las caracteristicas macroscópicas de longitud del vellón y/o libris. color, sedosidad, rizado. conformación del vellón, uniformidad y sensación al laco; permilen clasificar las fibras entre especies o grupos de amimales dentro de una misma especic.

Las características microscópicas permiten una identificación a través de la observación cuidadosa de las caracteristicas histológicas que son diferenciadorasentre libras, sobre la base de palronescomo: cl diámetro y su regularidad a lo lareo de la libra, carackerísticass de sus márgeness o bordes, el tipo do médula si está presente, del grosor de la cutícula y la distribución y coloración de los gránulos de pigmentos en la cortera.

Fon linción de los parámetros anteriormente senalados. se presenta un cuadro resumen con los resultados de los restos arqueofaunísticos del silio Topalcr. Sislos indican una gran proporción de presencia de llamas y que en un contexto general pertenecen a libras de las palas, dado su grosor y escasa longitud ( 0,5 a $1 \mathrm{~cm}$.). Tambićn sc observan algunas mucstras de guanaco o vicuñas, en yue no es posiblo diferenciarlas por su grato de deterioro, pero perfectamente diferenciables de alpaca o llama. Hay 4 muestas que pertenecen a vellones de guamaco y 2 de zorro, asi como 5 muestras obtenidas de un cucro de puma.

Jinalmente cahe señalar que si bien el análisis lanimérico es un buen complemento en lat identilicación de fibras de distintas especies y gue por su eslatlo de conservación permite tabajar eon mucstras argueológicas, defmitivamente no es confiable en la búsqueda de indicadores para sexo, edad o función rootécnicat.

\begin{tabular}{|c|c|c|c|c|}
\hline \multirow{2}{*}{$\begin{array}{c}\text { N"IDE } \\
\text { MUESTRA }\end{array}$} & \multirow[b]{2}{*}{ IDLATIIICACION } & \multicolumn{2}{|l|}{ IXX MLN I $A$ NIMEIRICO } & \multirow[b]{2}{*}{ ESIECIE } \\
\hline & & MACROSCOPICO & MICKOSCOIICO & \\
\hline $1-2$ & Top $\mathrm{H7}$ & $\begin{array}{l}\text { Poloticso. trenzardo y cale } \\
\text { oscuro. }\end{array}$ & 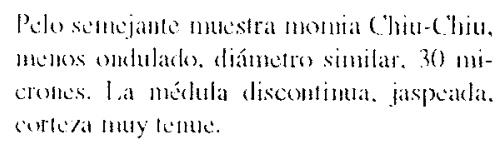 & Pololnumano \\
\hline $\begin{array}{l}3 \\
4 \\
5 \\
6 \\
7\end{array}$ & 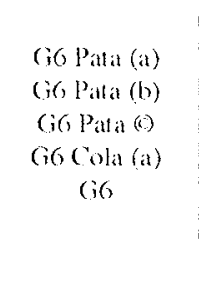 & 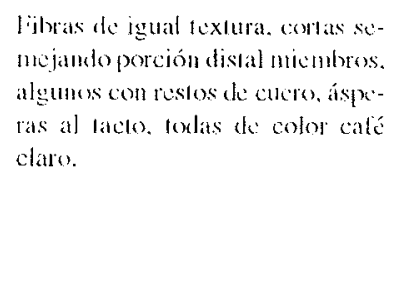 & 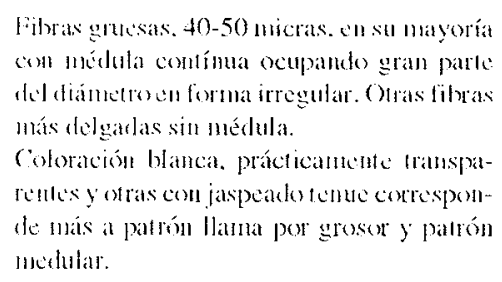 & I. lama \\
\hline $\begin{array}{c}8 \\
9 \\
10^{*}\end{array}$ & $\begin{array}{l}\text { M5 } \\
\text { MS (palas) } \\
\text {.6 }\end{array}$ & 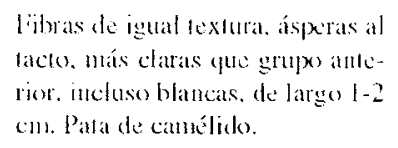 & 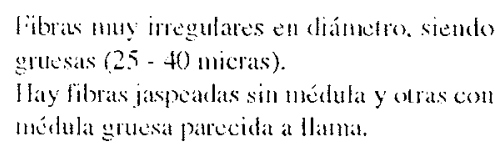 & Hama? \\
\hline 11 & D) 3 & 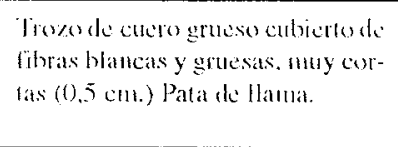 & 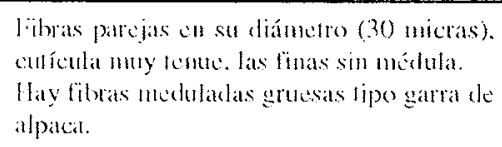 & Apaca? \\
\hline
\end{tabular}




\begin{tabular}{|c|c|c|c|c|}
\hline & & ISXAMEN IANIMETRICO & & \\
\hline $\begin{array}{c}N \text { DE } \\
\text { MLASTRA }\end{array}$ & DENTIRTCACION & MACkoscorleo & MICROSCOHCO & FSPECCE \\
\hline 12 & M7 (a) & $\begin{array}{l}\text { Pelo suctto largo enredarlo. cals } \\
\text { oscuro. ídem } 1 \text {. }\end{array}$ & lakm & Petoliunano \\
\hline 13 & $M 7(b)$ & $\begin{array}{l}\text { Vellón largo }(7-8 \text { cm. }) \text { clarobasey } \\
\text { puntas intactas. sin brilte aspetas. } \\
\text { tiesa. No puede ser patio. }\end{array}$ & 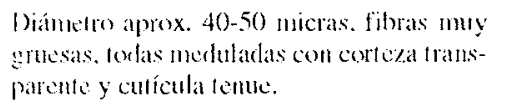 & L.lanm? \\
\hline $\begin{array}{l}14 \\
15 \\
18\end{array}$ & $\begin{array}{l}\text { Top } 06 \\
\text { Top } 006 \\
118\end{array}$ & $\begin{array}{l}\text { Mibras muy cortas }(0.5 \mathrm{~cm} \text { ) con } \\
\text { restos de cuefo café osuro, foscas } \\
\text { y duas al tacto. Pata di thana. }\end{array}$ & 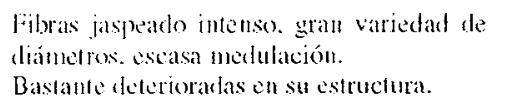 & LAama? \\
\hline 16 & L9: & $\begin{array}{l}\text { Filmas oscuras. largo } 2,3 \mathrm{~cm} . A s- \\
\text { peras. }\end{array}$ & $\begin{array}{l}\text { libras negras gruesas } 3.5-40 \text { micras, no se } \\
\text { distinguen estructuras. }\end{array}$ & $\ldots \ldots$ \\
\hline 17 & $1.9 \mathrm{~b}$ & $\begin{array}{l}\text { Fibras largas aisladas. áperas al } \\
\text { latco. café sin brillo. }\end{array}$ & $\begin{array}{l}\text { Pibtas nuy gruesas } 70-80 \text { micras aislatlas, } \\
\text { caté oscuro sin mélula. }\end{array}$ & 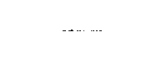 \\
\hline $\begin{array}{l}19 \\
20 \\
21 \\
22 \\
23 \\
24\end{array}$ & 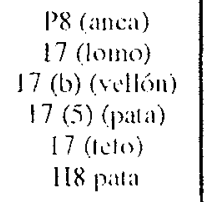 & 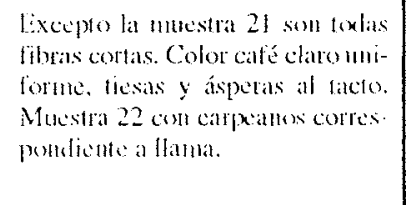 & $\begin{array}{l}\text { Whats gruesas } 40-50 \text { micras, mérula cont inta } \\
\text { con frecuencia y dispareja en su diametro. }\end{array}$ & l. lauma \\
\hline 25 & D) 3 & $\begin{array}{l}\text { Vellón conto con fibras sedosas y } \\
\text { aspecto tanoso con fibras gruesas. } \\
\text { liesas, café oscuro. No comespon- } \\
\text { dé at patat. }\end{array}$ & 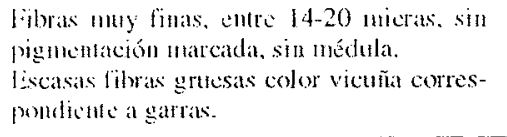 & $\begin{array}{l}\text { Gunntuco? } \\
\text { Vicuña? }\end{array}$ \\
\hline 26 & $N 6$ & 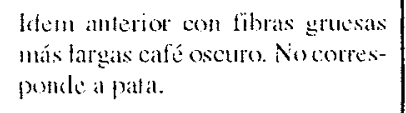 & Itemantrior. & $\begin{array}{l}\text { Guantaco? } \\
\text { Vicuña? }\end{array}$ \\
\hline $\begin{array}{l}27 \\
28 \\
20 \\
31\end{array}$ & $\begin{array}{l}\text { P8 (lomo) } \\
\text { M5 (lomo) } \\
\text { M5 (a) } \\
\text { M5 (b) }\end{array}$ & $\begin{array}{l}\text { Vollones con fibras finas, contas y } \\
\text { gruesas, targas y oscuras. No per } \\
\text { lemecon a pata, sedoso. }\end{array}$ & 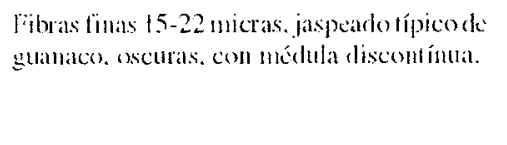 & Guantaco \\
\hline $\begin{array}{l}30 \\
33 \\
34 \\
35\end{array}$ & $\begin{array}{l}\text { Ha (pala) } \\
\text { 103 } \\
\text { M5(d) } \\
\text { (i) }\end{array}$ & 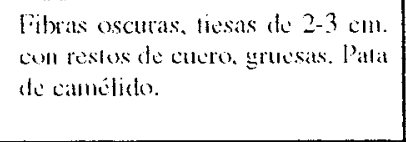 & Jden conjunto muestras 19 a 24. & L.ama \\
\hline $\begin{array}{l}32 \\
40\end{array}$ & $\begin{array}{l}18(b) \\
1.9\end{array}$ & $\begin{array}{l}\text { lïbras grtesas color calé rojizo } 2- \\
3 \mathrm{~cm} \text {. longitud. }\end{array}$ & 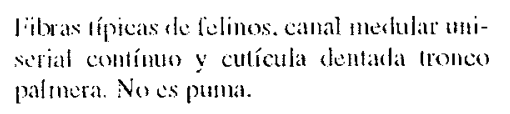 & Torro \\
\hline $\begin{array}{l}36 \\
37\end{array}$ & $\begin{array}{l}A 3(a) \\
A 3(b)\end{array}$ & $\begin{array}{l}\text { lilwas largas gmesas, tiesas blan- } \\
\text { cas y megrats mezeladas antifictal- } \\
\text { menle. }\end{array}$ & 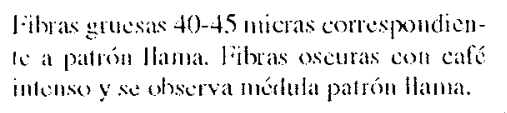 & Llama \\
\hline 38 & $D \cdot 3(\operatorname{lom} s)$ & $\begin{array}{l}\text { Veflón con fibras finas y gruesas } \\
\text { largas calé ascuro. No correspon- } \\
\text { de a pata. }\end{array}$ & 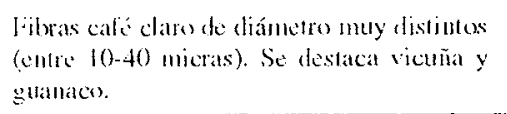 & $\begin{array}{l}\text { Llama? } \\
\text { Alpatcat? }\end{array}$ \\
\hline $\begin{array}{l}39) \\
41\end{array}$ & $\begin{array}{c}16(\mathrm{~b}) \\
118(\mathrm{pata})\end{array}$ & 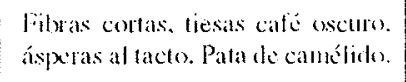 & $\begin{array}{l}\text { Vibas muy cortas y muy linas } 6-15 \text { micris y } \\
\text { con catacterísticats de patrón vicuña. lata? }\end{array}$ & Vicuña \\
\hline 42 a 46 & H8 Top & $\begin{array}{l}\text { litbras cortas tiesas y en baja cami- } \\
\text { dacl. (mucsta oblenisla distintas } \\
\text { gonas cuero puma). }\end{array}$ & 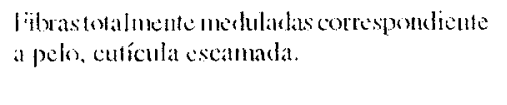 & Puma \\
\hline
\end{tabular}

* Este trabajo fue patrocinado y financiado por el Proyecto lOONDECYT 1950346. 


\section{BIBLIOGBRAFIA}

ADARO, L. y BINA VIENTE: M. A. Identificación 1998 de indicadores en el escueleto axil de camélidos sudamericanos. Avances de Ciencias Veterinarias (Santiago) $N^{\prime \prime} 7, p p .27-3.5$.

ARIAS, (i.; BIENAVINTI: M. A. Y GIECWLI, Pli1094 nio. "lentiticación y variabili-dad del uso del animal a través de lextiles arquoológicos: contrasic con patrones actuales". Actas del XII Congreso Nacional de Arqueología Chilema, l'cmuco, pp. $155-162$.

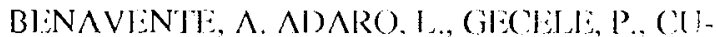
1993 NAYZA, (C. Contribución a la determinación de especies animales en arquenlogía: Fami-lia Camelidae y Taruca del Nor-te. Samtiago. (1. De (hile. D'ti.

BLNAVENTE: M. A. y ADAR( ). L. "Sclección de 1991 algumos indicadores óscos actuaIes de la familia Canclidac sudamericana y su contraste con muestras arqueológicas". Revista Chilena cle Anatomía, (Santiago) vol. 1, fitsc. 1, p. 34 .

BISNAVIENTL: M. А. "Determinación de especies 1094 de camb́liclos sudamericanos. Un cnfocpuczooarcucológico". Revista Chilena de Antropología. Sanliago. Iniversidad de Chile, $\mathrm{N}^{\circ}$ II. pl. $+\mid-5 I$.
BLNAVIENTI, A.: ADARO, L.: GEClELL, P. Aná1094 lisis de textiles e indicadores de fibras del sitio de Topater. M.S.

BI:N^ VI:NTL, M. ^. Chiu-Chiu 200: Un campa1981 mento de pastores. Santiago, Universidad de Chile, (Tesis).

BROTHWIII, K. J. And 1). HIGGS. Ciencia en 1982 Arqueología, México, ICLE.

C.ANAVOS, (i. Probabilidad y Estadística. Apli1988 caciones y Métodos. McGrawIHill Lispaña.

(.ARIDO) 1054

( AR'IAIENA, I: BLNAVIENTIE, M. A.: (BI:CLLLE, P.:CONCHA, I. y BI:NA VENTE, J. M. The transport function in camelids: an archacoroological approach. M.S.

DAVIS, S. The archaeology of animals. Lon1987 don, B. T. Bats ford.

MIENI:CA\%, A.: SAI,AMNI:, M. y ORTL\%, L. Una 1988 propuesta de sistematización de los caracteres morfoétricos los metapedios y falanges de Camélidos. Buenos Aires (inćd. 9)

( $A N \wedge \vee O S, G$. Probahiliclad y Listadística. Apli1988 cacionesy Métodos McGraw-Hill Expaña.

SCIIIFFE, H. The Analysis of Variance. Wiley 1950 Now York. 